\title{
Reaction of Bis(pinacolato)diboron with H-Si(100): The Pursuit of On- surface Hydrosilane Borylation Reactions
}

\author{
Esther Frederick, ${ }^{* 1}$ Igor Kolesnichenko, ${ }^{1}$ Quinn Campbell, ${ }^{1}$ Luis Fabián Peña, ${ }^{1}$ Angelica Be- \\ navidez, ${ }^{2}$ Evan M. Anderson, ${ }^{1}$ David R. Wheeler, ${ }^{1}$ Shashank Misra*1
}

AUTHOR ADDRESS ${ }^{1}$ Sandia National Laboratories, Albuquerque, NM, 87185, ${ }^{2}$ University of New Mexico, Albuquerque, NM, 87131

\begin{abstract}
On-surface solution phase chemical reactions, which are inherently amenable to scale-up, provide a pathway towards overcoming challenges present in gas phase processes for ultradoping of $\mathrm{Si}$, a process that introduces unprecedented concentration of dopant. Ultradoping, which can only be achieved with a direct chemical bond between dopant and Si, fundamentally changes the electronic properties of $\mathrm{Si}$, making it a promising next-generation electronic material. Traditional processes for solvent-based chemical functionalization attach species to the Si surface through carbon or oxygen linkers, which limits activated dopant density. This prevents solution phase chemistry from being useful for applications involving ultradoped Si. Recent work has focused on forming a direct on-surface Si-dopant bond to provide a scalable ultra-doping pathway. In this work, we expand upon that goal by demonstrating that well-known homogeneous chemistries can be usefully applied to surface reactions for ultradoping Si. By adapting a hydrosilane borylation reaction used to synthesize silyl boranes into a surface chemistry reaction, we successfully incorporate $1.3 \mathrm{e} 14 \mathrm{~cm}^{-2} \mathrm{~B}$ using scalable on-surface solvent-based chemistry. This density is high enough to produce the overlap of dopant wavefunctions required for achieving unprecedented conductivity in Si. Using computational studies, performed with the assumption that catalyst interaction was negligible, we predict the reaction straightforwardly occurs through a Si-B bond. However, with extensive experimental characterization including infrared spectroscopy, x-ray photoelectron spectroscopy, and secondary ion mass spectroscopy we elucidate cross-reactivity between the substrate, Bis(pinacolato)diboron and catalyst. The reaction complexity indicates that radical initiating catalysts are not benign in surface chemistry systems.
\end{abstract}

\section{INTRODUCTION}

Miniaturization of feature sizes is a driving force in the semiconductor industry and has been key in enabling small, low-cost, high-powered computing devices. As feature sizes shrink, new materials challenges are being broached to achieve the benchmarks set by the International Roadmap for Devices and Systems. ${ }^{1}$ One promising method, called atomic precision advanced manufacturing (APAM), utilizes ultra-high vacuum (UHV) based chemistry to form direct silicon (Si)-dopant bonds. This direct bond enables dopants to be incorporated as an ultra-thin layer containing an unprecedented dopant concentration (ultra-doping) in $\mathrm{Si}$ (e.g. phosphorus 2D density of $2 \mathrm{e} 14 \mathrm{~cm}^{-2}$ and 3D density of $1 \mathrm{e} 22$ $\left.\mathrm{cm}^{-3}\right)_{.2,3}$ The electronic behavior of $\mathrm{Si}$ is fundamentally changed by ultra-doping, as it causes the band structure of the material to be determined by the electrons confined in the dopant layer rather than by the properties of the parent material. This behavior is believed to be due to overlapping electronic structures of the confined dopants which allow coherent electronic transport from dopant to dopant without having to interact with the host material. ${ }^{4}$ These promising UHV results have limited utility due to a lack of scalability. ${ }^{3,5}$ Solution phase procedures, which are inherently amenable to scale-up, provide a pathway to scalable ultradoping, however the current chemistries are limited by methods that require a high temperature anneal for dopant activation, restricting their incorporation into traditional semiconductor fabrication processes. In this work, we addressed this limitation by adapting a well-known homogenous synthetic chemistry hydrosilane borylation reaction, into an on-surface chemical reaction to provide a route for directly functionalizing $\mathrm{Si}(100)$ with dopant containing species.

Literature reviews ${ }^{6-8}$ concisely summarize the wet chemical pathways previously used to achieve doping. Self-assembled monolayers (SAMs) as a standalone material have a high degree of versatility and inherent patternability that make them a promising material for engineering future devices. ${ }^{9,10}$ Molecular monolayer doping (MLD), uses dopant containing SAMs to produce ultra-shallow $(<5 \mathrm{~nm})$ doping of B (concentration $10^{19}-10^{20}$ atom $/ \mathrm{cm}^{-3}$ ) in $\mathrm{Si}(100)$ via solution phase chemistry. ${ }^{11,12}$ In MLD, dopant containing monolayers are chemically attached to an $\mathrm{H}-\mathrm{Si}(100)$ substrate via a $\mathrm{Si}-\mathrm{C}$ or $\mathrm{Si}-\mathrm{O}$ bond, followed by a requisite high temperature anneal $\left(\sim 1000^{\circ} \mathrm{C}\right)$ to decompose the precursor molecules, drive the dopant into the underlying $\operatorname{Si}(100)$ and activate it. Updates to the MLD capping process include using alternatives to caps, such as silsesquioxanes, ${ }^{13}$ spinon-glass, ${ }^{14}$ and a sacrificial SAM coated substrate. ${ }^{15,16}$ MLD has also been explored for other resists such as oxides, which helps minimize contamination from non-dopant species. ${ }^{17-21}$ While solution phase processing allows for scalability, the high temperature anneal required for dopant incorporation limits the utility of wet chemistry methods as it limits dopant concentration to its solid solubility in $\mathrm{Si}$ and 
places restrictions on incorporating ultra-doping into traditional semiconductor fabrication processes.

To obtain scalable routes to the transformational Si electronic behavior seen in APAM, we need solution phase chemistry processes that work without an anneal, namely processes involving direct Si-dopant chemistries. Recent work has focused on forming a direct Si-dopant bond by reacting boron trichloride with hydrogen and chloride terminated $\mathrm{Si}(100) .{ }^{22}$ In this paper, we expand upon that work with a proof-of-principle demonstration showing that wellknown homogeneous chemistries can be usefully applied to surface reactions for ultradoping Si. To do this, we adapted a solution phase hydrosilane borylation reaction ${ }^{23}$ that utilizes iridium as a radical initiating catalyst to form silylboranes from alkyl functionalized monohydrosilanes and bis(pinacolato)diboron $\left(\mathrm{B}_{2} \mathrm{Pin}_{2}\right)$ (Figure 1). As this homogeneous reaction works with sterically hindered $\mathrm{Si}$ species, we hypothesized this would work in surface reactions which inherently have steric restrictions. Additionally, the pinacol ligand was expected to help circumvent the well-known instability of the Si-B bond to ambient moisture. ${ }^{24}$ Since metals are undesirable for electronic applications, we substituted the Ir with the organic radical initiating catalyst azobisisobutyronitrile (AIBN). We show that this process yields a high concentration of incorporated $B$, though the radical initializing catalyst is not benign. This enables us to establish initial rules for successful choice of precursors and catalysts in on surface hydrosilane borylation reactions.

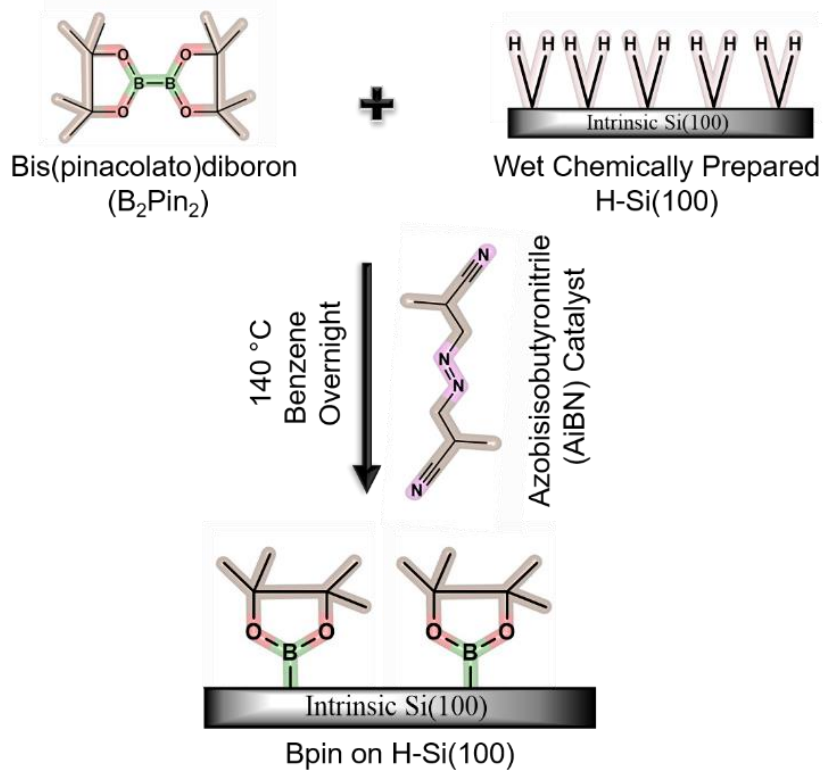

FIGURE 1 Proposed reaction of $\mathrm{B}_{2} \mathrm{Pin}_{2}$ with $\mathrm{H}-\mathrm{Si}$ using the radical initiating catalyst, AIBN. We adapted this on-surface reaction from a traditional solvothermal hydrosilane borylation reaction, which demonstrated Si-B formation using Iridium as a radical initiating catalyst. ${ }^{23}$

\section{RESULTS AND DISCUSSION}

The chemical reaction we used in this work is based on a solution phase homogenous hydrosilane borylation reaction. To adapt to an on-surface reaction, we performed the setup in an Ar glovebox using high purity precursors Bis(pinacolato)diboron ( $\mathrm{B}_{2} \mathrm{Pin}_{2}$, Sigma Aldrich, 99\%) and Azobisisobutyronitrile (AIBN, Sigma Aldrich, 99\%), and anhydrous benzene. The reaction was run at $140^{\circ} \mathrm{C}$ overnight in an Ar environment, followed by sonication in anhydrous benzene (also performed under Ar). The latter was done as a cleaning step to remove all physisorbed species as evidenced by lack of any visible residue on the surface. We characterized the surface post-reaction using a combination of computation, x-ray photoelectron spectroscopy (XPS), secondary ion mass spectroscopy (SIMS), and infrared spectroscopy (IR). A detailed description of the synthesis and characterization can be found in the SI.

We confirmed the successful attachment of $\mathrm{B}_{2} \mathrm{Pin}_{2}$ to the surface using SIMS (Figure 2). In order to demonstrate that we successfully surpassed the B concentration needed to enable the transformational Si behavior, we compared SIMS values to well-quantified values of the dopant concentrations needed for wave function overlap. The SIMS B density was $1 \mathrm{e} 20 \mathrm{~cm}^{-3}$ and the integrated B sheet density was $1.3 \mathrm{e} 14 \mathrm{~cm}^{-2}$. These densities are indicative of a very strong overlap of the wavefunctions on adjacent dopants, a requirement to see the unusual electronic properties of ultradoping. Assuming a full monolayer coverage of B corresponds to a surface site density of $7.3 \mathrm{e} 14 \mathrm{~cm}^{-2}$, our results corresponded to $\sim 18 \%$ monolayer coverage of $\mathrm{B}$.

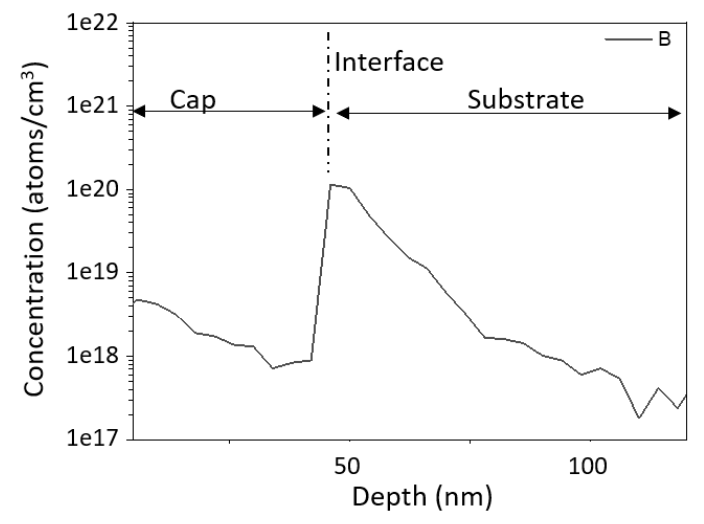

FIGURE 2 SIMS B data post $\mathrm{B}_{2} \mathrm{Pin}_{2}$ reaction showed the on-surface hydrosilane borylation reaction deposited $1.2 \mathrm{e} 20 \mathrm{~cm}^{-3}$ of $B$ at the interface ( $\sim 50 \mathrm{~nm}$ depth). The top $\sim 50 \mathrm{~nm}$ were a Si epi cap deposited after the on-surface reaction. The width of the trailing edge of the profile is a well-known measurement artifact from the sputtering of light species in silicon, and the density of atoms is expected to occur in a thin sheet. The corresponding integrated B sheet density was $1.3 \mathrm{e} 14 \mathrm{~cm}^{-2}$.

Having successfully confirmed we attached a technologically useful amount of B to the surface, we set about elucidating whether we had achieved a direct Si-dopant bond using a combination of computational and experimental studies. Density functional theory (DFT) calculations predicted the reaction should proceed as desired, with the $\mathrm{B}_{2} \mathrm{Pin}_{2}$ reacting with the Si substrate, and bonding preferentially through the B species. A detailed description of the computational methods is available in the SI. As a starting point for calculations, we assumed the catalyst strips the hydrogen resist from the silicon. We also assumed the catalyst generated a BPin fragment, with an exposed boron radical reacting with the bare silicon substrate. We then calculated the adsorption energies of binding the BPin fragment to the bare Si through the oxygen species and through the boron 
species and compared the two values to determine if there was a preference. To minimize the computational complexity of our problem we investigate a truncated BPin fragment, where the boron-oxygen-carbon ring remains intact, but the outlying methyl groups are instead truncated with hydrogen. Since the focus of our investigation is on determining the most likely bonding route for a Bpin fragment through either boron or oxygen, this truncation is highly unlikely to affect the result.

The computational results indicated that Bpin fragments are favored to bond directly to the silicon via the boron atom, as shown in Fig. 3. We predicted an adsorption energy of -3.61 eV for the Bpin fragment adsorbing through the boron atom and an adsorption energy of $-1.16 \mathrm{eV}$ for the Bpin fragment adsorbing by bonding through one of the oxygen atoms in the surrounding ring. While adsorption is thermodynamically favorable through either route, the boron route is nearly $2.5 \mathrm{eV}$ more thermodynamically favorable, providing a strong driving force for Bpin to directly bond to Si through boron. We found no kinetic reaction barrier to the direct adsorption of either of these configurations. It should be noted that our calculations were performed in vacuum and do not include any interaction with the solution or catalyst. While the computational system does not fully reflect experimental conditions, the effects of these interactions are often assumed to be negligible. With the assumption that the catalyst and solution do not play a significant role in the evolution of the system, the computation strongly indicated that $\mathrm{B}_{2}$ pin 2 directly through boron as desired.

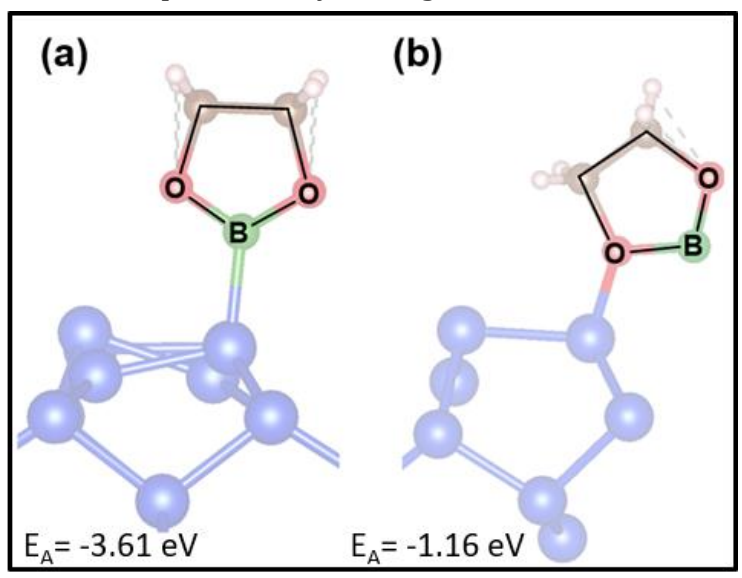

FIGURE 3 A side view of the calculated configurations for the truncated Bpin fragment bonding to the silicon surface through (a) boron and (b) oxygen. While adsorption is thermodynamically favorable through either route, the boron bonded BPin is nearly $2.5 \mathrm{eV}$ more thermodynamically favorable, providing a strong driving force for Bpin to bond to Si through boron.

Having computationally predicted that the reaction would proceed with an Si-B bond, we turned to a combination of XPS and IR to determine if we succeeded in experimentally achieving a direct Si-B attachment. The XPS data of the sample exhibited a peak in the B 1s region at $192.5 \mathrm{eV}$, which matched published data for B-O species. ${ }^{32,33}$ The XPS B 1s region did not elucidate the $B$ attachment, since regardless of surface attachment ( $\mathrm{Si}-\mathrm{B}-\mathrm{O}$ or $\mathrm{Si}-\mathrm{O}-\mathrm{B}$ ), the $\mathrm{B}$ is attached to an 0 species. $^{34}$ We also used the $B$ region to assess if the pinacol group enhanced the stability of the B. After 8 days in ambient storage, B-O was no longer visible, indicating the pinacol group did not significantly stabilize the B (See SI Figure 1). To further investigate the B attachment, we analyzed the Si $2 p$ regions of both the sample and control (Figure 4). We performed an additional calibration to align the Si $2 \mathrm{p}_{3 / 2}$ peaks to the same value $(99.02 \mathrm{eV})$. Both $\mathrm{Si} 2 \mathrm{p}$ regions show a single higher energy peak, at $102.55 \mathrm{eV}$ in the control and $102.39 \mathrm{eV}$ in the $\mathrm{B}_{2} \mathrm{Pin}_{2}$ sample. In the control, where no $\mathrm{B}$ is expected, this higher energy peak was unambiguously assigned to the $\mathrm{SiO}_{2}$ species. In the sample, we could not confidently assign the $102.39 \mathrm{eV}$. On the one hand, this could be interpreted as an $\mathrm{Si}-\mathrm{B}$ bond. The peak matched known Si-B bond values of $\sim 102 \mathrm{eV},{ }^{34}$ and the $-0.16 \mathrm{eV}$ shift of the peak relative to the control could be attributed to the lower electronegativity of B relative to 0. Additionally, the higher \% Si $2 \mathrm{p}$ area of this peak in the sample(16.4\%) versus the control $(14.1 \%)$, suggested that the sample peak is a combination of $\mathrm{SiO}_{2}$ and $\mathrm{Si}-\mathrm{B}$ species. On the other hand, this small shift could also be attributed to differences in chemistry of neighboring species ${ }^{35}$, in other words, the presence of other species on the surface from introducing the $\mathrm{B}_{2} \mathrm{Pin}_{2}$ could have affected the oxide position. Due to the similarity in position of the peaks in the control and sample, we could not confidently assign the $102.39 \mathrm{eV}$ peak in the sample. Since XPS did not provide conclusive experimental evidence of the bonding configuration for the reaction, we turned to IR to see if we could get further information about the surface chemistry.

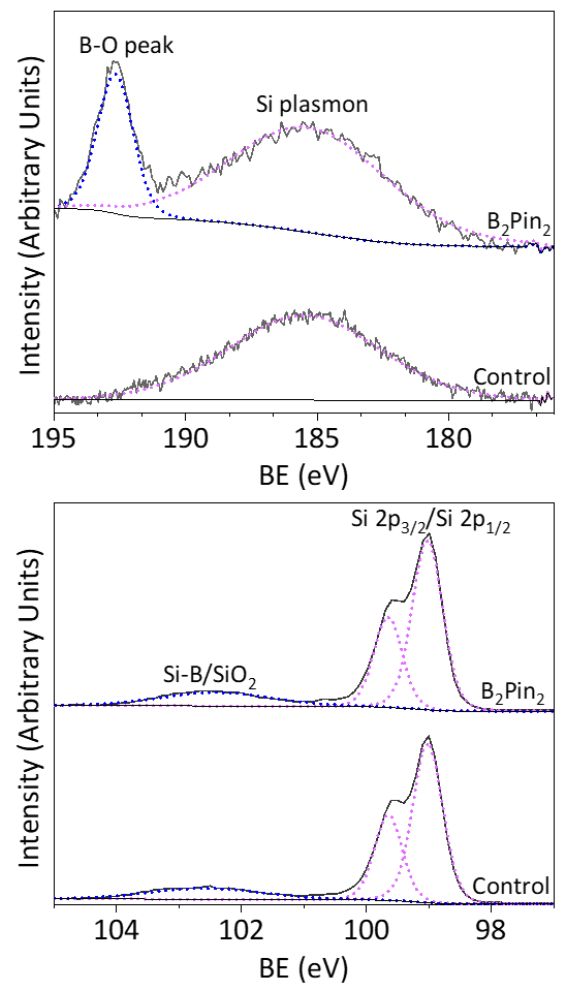

FIGURE 4 TOP: XPS data of the $B$ 1s region in the sample (B2Pin2/AIBN) and control (AIBN only). In the B 1s region, only $\mathrm{B}_{2} \mathrm{Pin}_{2}$ showed a B-O peak. BOTTOM: XPS data of the Si $2 \mathrm{p}$ regions of the sample and control. Both displayed a broad peak between $102-104 \mathrm{eV}$. Due to the similarity in $\mathrm{Si}-\mathrm{B}$ and $\mathrm{SiO}_{2}$ $\mathrm{BEs}$, we could not confidentially assign the sample peak.

The IR fingerprint region suggested that the $\mathrm{B}_{2} \mathrm{Pin} 2$ did not bind through 0 , substantiating the computational results. 
However, the lack of an Si-B peak left us without conclusive evidence of a Si-B bond. Using DFT, we predicted the IR spectra for $\mathrm{B}_{2} \mathrm{Pin}_{2}, \mathrm{~B}_{2} \mathrm{Pin}_{2}-\mathrm{O}-\mathrm{SiH}_{3}$ and $\mathrm{B}_{2} \mathrm{Pin}_{2}-\mathrm{SiH}_{3}$. The agreement of the computed $\mathrm{B}_{2} \mathrm{Pin}_{2}$ spectrum (See SI figure 2) with the published spectrum, ${ }^{36}$ validated the computational methods. A detailed description of the computational method is available in the SI. By comparing the spectra with each other and known literature values, we identified characteristic peaks for $\mathrm{B}-\mathrm{Si}$ from the $\mathrm{B}_{2} \mathrm{Pin}_{2}-\mathrm{SiH}_{3}$ and $\mathrm{B}-\mathrm{O}-\mathrm{Si}$ from the $\mathrm{B}_{2} \mathrm{Pin}_{2}-\mathrm{O}-\mathrm{SiH}_{2}$ calculation which assumes adventitious oxygen either from air or moisture and provides a B-O peak distinctive from the $\mathrm{B}-\mathrm{O}-\mathrm{C}$ in the $\mathrm{B}_{2} \mathrm{Pin}_{2}$. and $\mathrm{B}-\mathrm{Si}$ from the $\mathrm{B}_{2} \mathrm{Pin}_{2}-\mathrm{SiH}_{3}$ (Figure 5). ${ }^{37-39}$

We used the calculated peaks to analyze the experimental differential IR absorbance spectra (Figure 5) of a Si-H surface, a control ( $\mathrm{H}-\mathrm{Si}$ heated with solvent/AIBN), and a $\mathrm{B}_{2} \mathrm{Pin}_{2}$ sample. The B-O peak at $1482 \mathrm{~cm}^{-1}$ was not seen, which strongly suggested the $\mathrm{B}_{2} \mathrm{Pin}_{2}$ was not binding through a B-O species. The fingerprint regions of all the samples below $1400 \mathrm{~cm}^{-1}$ look identical, displaying only the Si phonon peaks. The lack of an Si-B species at $1249 \mathrm{~cm}^{-1}$, could not be taken as conclusive evidence that there was not an Si-B bond due to significant overlap with strong Si phonons in that region. The main difference between the spectra was a $1448 \mathrm{~cm}^{-1}$ peak seen only in the AIBN spectrum, attributed to a $\mathrm{CH}_{2}$ species from AIBN. ${ }^{38}$ The diminishment of this peak in the $\mathrm{B}_{2} \mathrm{Pin}_{2}$ spectrum indicated the $\mathrm{B}_{2} \mathrm{Pin} 2$ reacted with surface species.
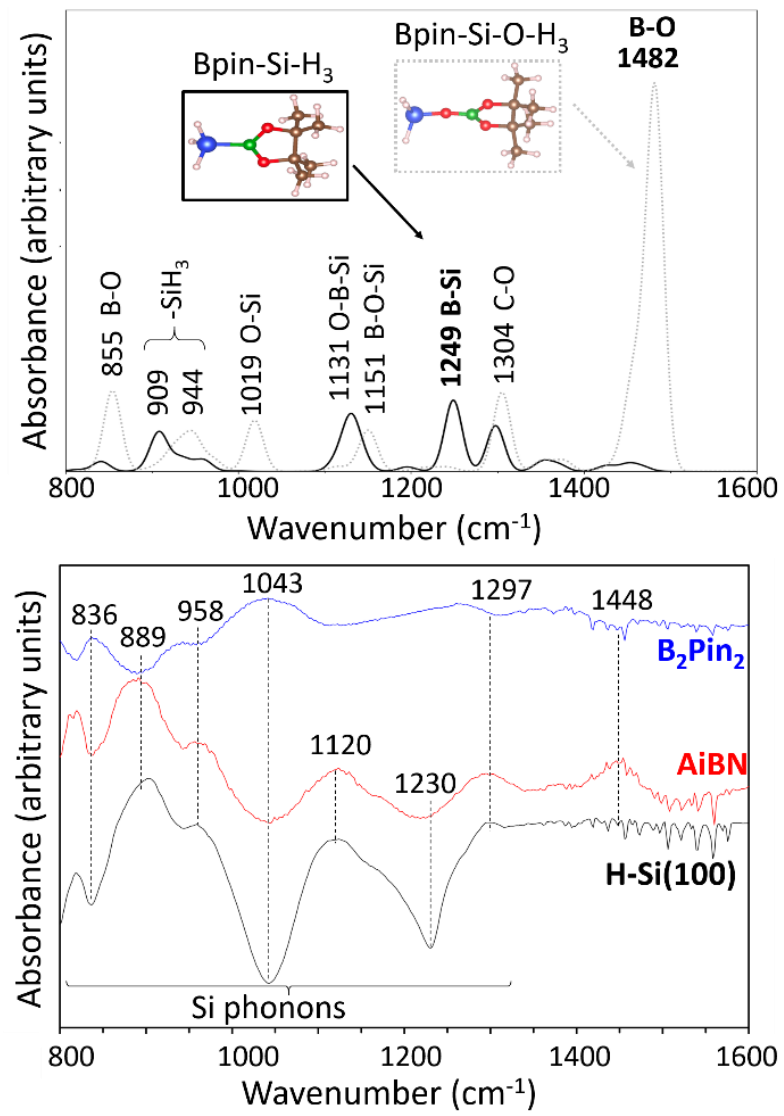

FIGURE 5 Top: Computational spectra of $\mathrm{B}_{2} \mathrm{Pin}_{2}-\mathrm{O}-\mathrm{SiH}_{2}$ and $\mathrm{B}_{2} \mathrm{Pin}_{2}-\mathrm{SiH}_{3}$, which respectively provided a distinguishing $\mathrm{B}-\mathrm{O}$ peak $\left(1482 \mathrm{~cm}^{-1}\right)$ and B-Si peak $\left(1249 \mathrm{~cm}^{-1}\right)$. Bottom: IR spectra of H-Si(100) (referenced to Si(100)), AIBN control (referenced to $\mathrm{H}-\mathrm{Si}(100)$ ), and $\mathrm{B}_{2}$ pin 2 sample (referenced to $\mathrm{H}-\mathrm{Si}(100)$ ). The flip in Si phonon absorbances in the $\mathrm{B}_{2} \mathrm{Pin}_{2}$ spectrum was attributed to thermal fluctuations from the glovebox purge. The lack of a B-O peak suggested $\mathrm{B}_{2} \mathrm{Pin}_{2}$ was not bound through a B-O-Si bond. The calculated B-Si peak was not observed but was potentially complicated by overlap with Si phonons.

We used SIMS, XPS and IR to obtain further insight into the catalyst involvement. Both SIMS and XPS showed a significant presence of $\mathrm{C}, \mathrm{O}$ and $\mathrm{N}$ on the surface (Figure 6). While we expected $\mathrm{C}$ and $\mathrm{O}$ from $\mathrm{B}_{2} \mathrm{Pin}_{2}$, the observed ratios in SIMS for C:B and O:B were $\sim 30: 1$, which was higher than expected ratios for $\mathrm{B}_{2} \mathrm{Pin}_{2}$ of $6: 1$ for $\mathrm{C}: \mathrm{B}$ and $2: 1$ for $0: \mathrm{B}$. In $\mathrm{XPS}$, the $\mathrm{N} 1 \mathrm{~s}$ region of the substrate after $\mathrm{B}_{2} \mathrm{Pin} 2$ reaction (Figure 6) had three distinct peaks at $402.6 \mathrm{eV}, 400.4 \mathrm{eV}$, and $397.9 \mathrm{eV}$; corresponding to the $\mathrm{C} \equiv \mathrm{N}, \mathrm{N}-(\mathrm{C}=0)$ and $\mathrm{N}-\mathrm{Si}$ species respectively. The $\mathrm{C}=0$ was further confirmed in the $\mathrm{C} 1 \mathrm{~s}$ region. The presence of $\mathrm{N}-\mathrm{C}$ and $\mathrm{N}-\mathrm{C}-\mathrm{O}$ species explained the unexpectedly high concentration of $\mathrm{C}$ and $\mathrm{O}$. As the AIBN catalyst was the only potential $\mathrm{N}$ source, this data confirmed the catalyst was not benign, and indicated AIBN reacted with the surface ( $\mathrm{Si}-\mathrm{N}$ species) as well as decomposed into species that underwent further oxidation $(\mathrm{N}-\mathrm{C}=\mathrm{O}$ species).
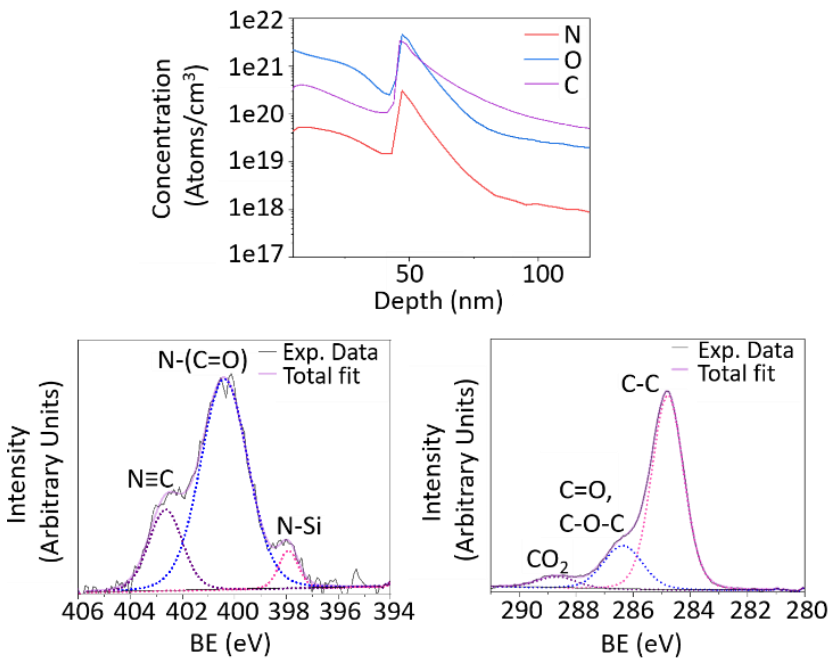

FIGURE $6 \underline{\text { Top: }}$ SIMS of the sample post $\mathrm{B}_{2} \mathrm{Pin}_{2}$ reaction had significant $\mathrm{C}, \mathrm{O}$ and $\mathrm{N}$. Bottom Left: $\mathrm{N}$ 1s XPS indicated AIBN bound to the surface $(\mathrm{Si}-\mathrm{N})$ and further reacted $(\mathrm{N}-\mathrm{C}=\mathrm{O})$. Bottom Right: C 1s XPS further confirmed the $\mathrm{C}-\mathrm{O}$ seen in the $\mathrm{N}-\mathrm{C}=\mathrm{O}$.

IR substantiated the conclusion that AIBN reacted with the surface and further demonstrated that the AIBN reacted with the $\mathrm{B}_{2} \mathrm{Pin}_{2}$ (Figure 7). After exposing an Si-H substrate to $\mathrm{B}_{2} \mathrm{Pin}_{2}$ or AIBN, the only difference in the spectra was a peak at $\sim 2260 \mathrm{~cm}^{-1}$ in the AIBN only spectrum, corresponding to $\mathrm{C} \equiv \mathrm{N}$. This indicated that in the presence of $\mathrm{B}_{2} \mathrm{Pin}_{2}$ the $\mathrm{C} \equiv \mathrm{N}$ group further oxidized into additional $\mathrm{C}=0$ species. As the AIBN and $\mathrm{B}_{2} \mathrm{Pin}_{2}$ systems had similar levels of adventitious $\mathrm{O}$ exposure, the only potential source of additional oxygen was the $\mathrm{B}_{2} \mathrm{Pin}_{2}$, thereby indicating the decomposition products of AIBN directly reacted with $\mathrm{B}_{2} \mathrm{Pin}_{2}$. 


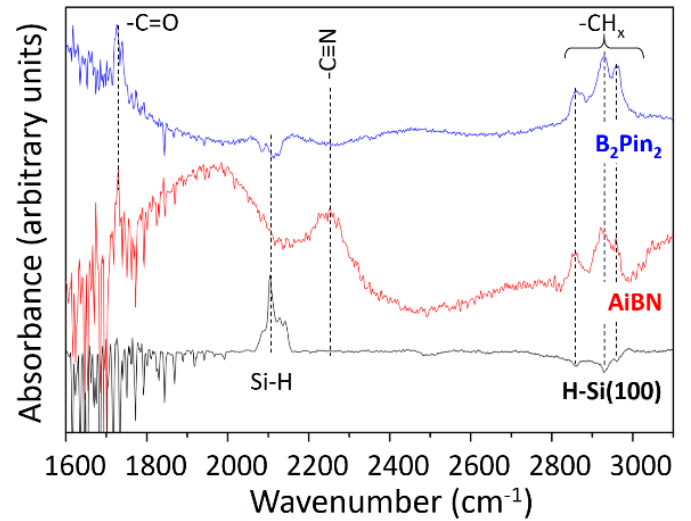

FIGURE 7 IR spectra of H-Si(100) (referenced to Si(100)), AIBN control (referenced to $\mathrm{H}-\mathrm{Si}(100)$ ), and $\mathrm{B}_{2}$ pin 2 sample (referenced to $\mathrm{H}-\mathrm{Si}(100))$. The IR data substantiated the AIBN crossreacted with $\mathrm{B}_{2} \mathrm{Pin}_{2}$ and the $\mathrm{H}-\mathrm{Si}(100)$ substrate.

From the consilience of SIMS, IR and XPS characterization, we determined that the bonding is more complicated than a straightforward singular Si-B bond and the catalyst actively reacted with both the surface and $\mathrm{B}_{2} \mathrm{Pin}_{2}$. This is important as radical initiating catalysts are known to most commonly proceed through decomposition reaction mechanisms, ${ }^{40}$ but common surface chemistry catalysts containing only $\mathrm{C}$ and 0 species, such as benzoyl peroxide, can be difficult to differentiate from solvent and precursor species using traditional characterization methods. The complexity introduced by cross-reactivity with AIBN resulted in an ensemble of chemistries and binding modes on the Si surface.

\section{CONCLUSION}

We successfully reacted $B_{2} P_{i n}$ with a $\operatorname{Si}(100)$ surface using AIBN as a radical initiating catalyst. Using SIMS, we determined we achieved $1.3 \mathrm{e} 14 \mathrm{~cm}^{-2}$ of $\mathrm{B}$, comfortably in the range needed to fundamentally change the electronic behavior of Si. The presence of B confirms that the $\mathrm{B}_{2} \mathrm{Pin}_{2}$ reacted with the underlying Si. Computational results, performed with the common assumption that the catalyst and solution interactions are negligible, predicted the bonding would occur directly through a Si-B bond. However, by using multiple characterization techniques of SIMS, IR and XPS, we determined that the bonding is more complicated than a Si-B bond alone. The presence of $\mathrm{N}$ and $\mathrm{C}=0$ confirmed the active participation of the AIBN as a reactant with both the surface and $\mathrm{B}_{2} \mathrm{Pin}_{2}$. The cross-reactivity of the catalyst led to a complicated surface chemistry resulting in an ensemble of binding modes.

Overall, while we demonstrated a method of attaching B to the surface, it is unclear if the adventitious chemistry will ultimately hinder electrical investigation. However, the high concentration of B is a proof-of-principle demonstration that solution phase on-surface chemistry can provide a scalable route towards the transformational Si behavior currently achieved using processes limited by ultra-high vacuum requirements. This opens up a pathway for using onsurface solution-based chemistry as a process for introducing dopants in future electronic devices. Our results demonstrate the continued need for synthetic chemists to adapt their knowledge of existing chemical processes in on-surface reactions. We will be investigating other chemical precursors and alternative catalysts, such as light and sonochemical activation, to achieve this goal and to establish further understanding of what chemistries we incur from these solution processes.

\section{ASSOCIATED CONTENT}

The Supporting Information is available free of charge at http://pubs.acs.org.

-Synthetic, Computational and Characterization Methods

-Figure SI 1 B XPS of $\mathrm{B}_{2} \mathrm{Pin}_{2}$ sample after $\sim 1$ week

-Figure SI 2 Computed $\mathrm{B}_{2} \mathrm{Pin}_{2}$ spectrum

\section{AUTHOR INFORMATION}

\section{Corresponding Authors}

Esther Frederick, Email: efreder@sandia.gov https://orcid.org/0000-0001-6363-1633

Shashank Misra, Email: smisra@sandia.gov

\section{Author Contributions}

The manuscript was written through contributions of all authors. All authors have given approval to the final version of the manuscript.

\section{ACKNOWLEDGMENT}

This work was supported by the Laboratory Directed Research and Development program at Sandia National Laboratories under project 213017 (FAIR DEAL) and performed, in part, at the Center for Integrated Nanotechnologies, an Office of Science User Facility operated for the U.S. Department of Energy (DOE) Office of Science. Sandia National Laboratories is a multi-mission laboratory managed and operated by National Technology and Engineering Solutions of Sandia, LLC, a wholly owned subsidiary of Honeywell International, Inc., for DOE National Nuclear Security Administration under contract DE-NA0003525. This paper describes objective technical results and analysis. Any subjective views or opinions that might be expressed in the paper do not necessarily represent the views of the U.S. Department of Energy or the United States Government.

\section{REFERENCES}

(1) IEEE. International Roadmap for Devices and Systems (IRDSTM), 2020 edition.

(2) Schofield, S. R.; Curson, N. J.; Simmons, M. Y.; Ruess, F. J.; Hallam, T.; Oberbeck, L.; Clark, R. G. Atomically Precise Placement of Single Dopants in Si. Phys Rev Lett 2003, 91.

(3) Ward, D. R. S., S.W.; Anderson, E.M.; Bussmann, E.; Tracy, L.; Lu, T.; Maurer, L.N.; Baczewski, A.; Campbell, D.M.; Marshall, M.T.; Misra, S. . Atomic Precision Advanced Manufacturing for Digital Electronics Electronic Device and Failure Analysis 2020, 22, 4 10

(4) Mazzola, F.; Chen, C.-Y.; Rahman, R.; Zhu, X.-G.; Polley, C. M.; Balasubramanian, T.; King, P. D. C.; Hofmann, P.; Miwa, J. A.; Wells, J. W. The Sub-band Structure of Atomically Sharp Dopant Profiles in Silicon. npj Quantum Materials 2020, 5, 34.

(5) Škereň, T.; Köster, S. A.; Douhard, B.; Fleischmann, C.; Fuhrer, A. Bipolar Device Fabrication Using a Scanning Tunnelling Microscope. Nature Electronics 2020, 3, 524-530.

(6) Holmes, J. O., J.; Duffy, R.; Long, B.: Surface Functionalization Strategies for Monolayer Doping. In Encyclopedia of Interfacial Chemistry, 2018; pp 829-834.

(7) Duffy, R. K., N.; Mirabelli, G.; Galluccio, E.; Hurley, P.K.; Holmes, J.D; Long, B. IEEE Review: Monolayer Doping and Other Strategies in High Surface-to-Volume Ratio Silicon

Devices. IEEE 2018. 
(8) O'Connell, J.; Biswas, S.; Duffy, R.; Holmes, J. D. Chemical Approaches for Doping Nanodevice Architectures. Nanotechnology 2016, 27

(9) Ulman, A. Formation and Structure of Self-Assembled Monolayers. Chem Rev 1996, 96, 1533-1554.

(10) Aswal, D. K.; Lenfant, S.; Guerin, D.; Yakhmi, J. V.; Vuillaume, D. Self Assembled Monolayers on Silicon for Molecular Electronics. Analytica Chimica Acta 2006, 568, 84-108.

(11) Ho, J. C.; Yerushalmi, R.; Jacobson, Z. A.; Fan, Z.; Alley, R. L.; Javey, A. Controlled Nanoscale Doping of Semiconductors via Molecular Monolayers. Nat Mater 2008, 7, 62-67.

(12) Ho, J. C.; Yerushalmi, R.; Smith, G.; Majhi, P.; Bennett, J.; Halim, J.; Faifer, V. N.; Javey, A. Wafer-Scale, Sub-5 nm Junction Formation by Monolayer Doping and Conventional Spike Annealing. Nano Lett 2009, 9, 725-730.

(13) Alphazan, T.; Mathey, L.; Schwarzwalder, M.; Lin, T. H.; Rossini, A. J.; Wischert, R.; Enyedi, V.; Fontaine, H.; Veillerot, M.; Lesage, A.; Emsley, L.; Veyre, L.; Martin, F.; Thieuleux, C.; Coperet, C. Monolayer Doping of Silicon through Grafting a Tailored Molecular Phosphorus Precursor onto Oxide-Passivated Silicon Surfaces. Chem Mater 2016, 28, 3634-3640.

(14) Gao, X. J.; Guan, B.; Mesli, A.; Chen, K. X.; Sun, L. M.; Dan, Y. P. Toward Defect-Free Doping by Self-Assembled Molecular Monolayers: The Evolution of Interstitial Carbon-Related Defects in Phosphorus-Doped Silicon. Acs Omega 2019, 4, 3539-3545.

(15) Hazut, O.; Agarwala, A.; Amit, I.; Subramani, T.; Zaidiner, S.; Rosenwaks, Y.; Yerushalmi, R. Contact Doping of Silicon Wafers and Nanostructures with Phosphine Oxide Monolayers. Acs Nano 2012, 6, 10311-10318.

(16) Ye, L.; Gonzalez-Campo, A.; Kudernac, T.; Nunez, R.; de Jong, M.; van der Wiel, W. G.; Huskens, J. Monolayer Contact Doping from a Silicon Oxide Source Substrate. Langmuir 2017, 33, 36353638.

(17) Longo, R. C.; Cho, K.; Hohmann, S.; Thissen, P. Mechanism of Phosphorus Transport Through Silicon Oxide During Phosphonic Acid Monolayer Doping. J Phys Chem C 2018, 122, 1008810095.

(18) Mathey, L.; Alphazan, T.; Valla, M.; Veyre, L.; Fontaine, H.; Enyedi, V.; Yckache, K.; Danielou, M.; Kerdiles, S.; Guerrero, J.; Barnes, J. P.; Veillerot, M.; Chevalier, N.; Mariolle, D.; Bertin, F.; Durand, C.; Berthe, M.; Dendooven, J.; Martin, F.; Thieuleux, C.; Grandidier, B.; Coperet, C. Functionalization of Silica Nanoparticles and Native Silicon Oxide with Tailored Boron-Molecular Precursors for Efficient and Predictive p-Doping of Silicon. J Phys Chem C 2015, 119, 13750-13757.

(19) Arduca, E.; Mastromatteo, M.; De Salvador, D.; Seguini, G.; Lenardi, C.; Napolitani, E.; Perego, M. Synthesis and Characterization of P Delta-layer in $\mathrm{SiO}_{2}$ by Monolayer Doping. Nanotechnology 2016, 27.

(20) Kohli, P.; Jain, A.; Chakravarthi, S.; Bu, H.; Dunham, S. T.; Banerjee, S. Interactions of B Dopant Atoms and Si Interstitials with $\mathrm{SiO}_{2}$ Films During Annealing for Ultra-shallow Junction Formation. J Appl Phys 2005, 97.

(21) van Druenen, M.; Collins, G.; Glynn, C.; O'Dwyer, C.; Holmes, J. D. Functionalization of $\mathrm{SiO}_{2}$ Surfaces for Si Monolayer Doping with Minimal Carbon Contamination. Acs Appl Mater Inter 2018, 10, 2191-2201.

(22) Silva-Quinones, D.; He, C.; Butera, R. E.; Wang, G. T.; Teplyakov, A. V. Reaction of $\mathrm{BCl}_{3}$ with $\mathrm{H}$ - and Cl-terminated $\mathrm{Si}(100)$ as a Pathway for Selective Monolayer Doping Through Wet Chemistry. Appl Surf Sci 2020, 533, 146907.
(23) Boebel, T. A.; Hartwig, J. F. Iridium-Catalyzed Preparation of Silylboranes by Silane Borylation and Their Use in the Catalytic Borylation of Arenes. Organometallics 2008, 27, 6013-6019.

(24) Noth, H.: Section 6.1.4 Metalloboranes. In Science of Synthesis; Kaufmann, D. E. M., D.S., Ed.; Georg Thieme Verlag: Germany, 2004; Vol. 6.

(25) QUANTUM-ESPRESSO Software Package. https://iopscience.iop.org/article/10.1088/0953-8984/21/39/395502.

(26) Perdew, J. P.; Burke, K.; Ernzerhof, M. Generalized Gradient Approximation Made Simple. Phys Rev Lett 1996, 77, 38653868.

(27) van Setten, M. J.; Giantomassi, M.; Bousquet, E.; Verstraete, M. J.; Hamann, D. R.; Gonze, X.; Rignanese, G. M. The PseudoDojo: Training and Grading a 85 Element Optimized Norm-conserving Pseudopotential Table. Computer Physics Communications 2018, 226, 39-54.

(28) Marzari, N.; Vanderbilt, D.; Payne, M. C. Ensemble Density-Functional Theory for Ab Initio Molecular Dynamics of Metals and Finite-Temperature Insulators. Phys Rev Lett 1997, 79, 1337 1340 .

(29) Monkhorst, H. J.; Pack, J. D. Special Points for Brillouinzone Integrations. Phys Rev B 1976, 13, 5188-5192.

(30) Aldinger, B. S.; Gupta, A.; Clark, I. T.; Hines, M. A. The same etchant produces both near-atomically flat and microfaceted $\operatorname{Si}(100)$ surfaces: The effects of gas evolution on etch morphology. J Appl Phys 2010, 107.

(31) NIST X-ray Photoelectron Spectroscopy Database, NIST Standard Reference Database Number 20, doi:10.18434/T4T88K. National Institute of Standards and Technology: Gaithersburg MD 20899, 2000.

(32) Hendrickson, D. N.; Hollander, J. M.; Jolly, W. L. Core-electron Binding Energies for Compounds of Boron, Carbon, and Chromium. Inorg Chem 1970, 9, 612-615.

(33) Bois, L.; L'Haridon, P.; Laurent, Y.; Gouin, X.; Grange, P.; Létard, J. F.; Birot, M.; Pillot, J. P.; Dunoguès, J. Characterization of a Boro-silicon Oxynitride Prepared by Thermal Nitridation of a Polyborosiloxane. Journal of Alloys and Compounds 1996, 232, 244-253.

(34) Bai, H. W.; Wen, G.; Huang, X. X.; Han, Z. X.; Zhong, B.; Hu, Z. X.; Zhang, X. D. Synthesis and Structural Characterization of SiBOC Ceramic Fibers Derived from Single-source Polyborosiloxane. Journal of the European Ceramic Society 2011, 31, 931-940.

(35) Bouvet, D.; Clivaz, P. A.; Dutoit, M.; Coluzza, C.; Almeida, J.; Margaritondo, G.; Pio, F. Influence of Nitrogen Profile on Electrical Characteristics of Furnace- or Rapid Thermally Nitrided Silicon Dioxide Films. J Appl Phys 1996, 79, 7114-7122.

(36) Spectral Database for Organic Compounds - SDBS. National Institute of Advanced Industrial Science and Technology.

(37) Coblentz Society, I.: In "Evaluated Infrared Reference Spectra" in NIST Chemistry WebBook, NIST Standard Reference Database Number 69, Eds. Linstrom, P.J.; Mallard, W.G.; National Institute of Standards and Technology, Gaithersburg MD 20899.

(38) Silverstein, R. M. W., F.X.; Kiemle, D.J. : Chapter 2: Infrared Spectrometry in Spectrometric Identification of Organic Compounds, Seventh Edition. John Wiley \& Sons: Hoboken, NJ, 2005.

(39) Ogata, Y. H.: Characterization of Porous Silicon by Infrared Spectroscopy. In Handbook of Porous Silicon; Canham, L., Ed.; Springer International Publishing: Cham, 2014; pp 473-480.

(40) Binkley, E. R. B., R.W. : II. Basic Stages of a Radical Chain Reaction, https://chem.libretexts.org/@go/page/23816. 2019. 
Authors are required to submit a graphic entry for the Table of Contents (TOC) that, in conjunction with the manuscript title, should give the reader a representative idea of one of the following: A key structure, reaction, equation, concept, or theorem, etc., that is discussed in the manuscript. Consult the journal's Instructions for Authors for TOC graphic specifications.
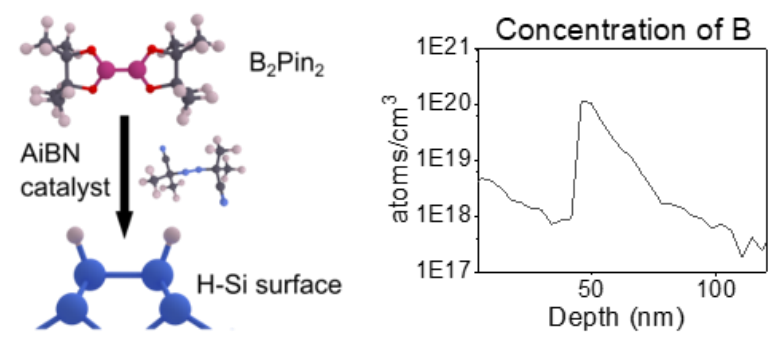

Insert Table of Contents artwork here 